\title{
Pricing Mechanisms for Multi-Carrier Wireless Systems
}

\author{
Anil Kumar Chorppath, Tansu Alpcan, \\ Technical University of Berlin \\ Deutsche Telekom Laboratories \\ Email: anil.chorppath@sec.t-labs.tu-berlin.de, \\ alpcan@sec.t-labs.tu-berlin.de
}

\author{
Holger Boche \\ Technical University of Munich \\ Munich, Germany \\ Email:boche@tum.de
}

\begin{abstract}
Multi-carrier wireless schemes and systems such as Orthogonal Frequency Division Multiplexing (OFDM), cognitive radios, and femtocells play an important role for efficient power and spectrum utilization. In uplink transmissions of these systems, independent and autonomous mobile users may act selfishly in order to get a higher share of available resources, which is encountered by a pricing mechanism that penalizes the interference they create. Specifically, the mobile users strategically decide on their power levels to minimize their cost which is the difference between their utilities based on Shannon capacity and payments. The base station acts as a mechanism designer to ensure that certain global objectives are satisfied when allocating resources. In this paper, we consider two such objectives: one is sum of user utility maximization and the other one is operator revenue maximization based on the prices charged to users, which coincidentally acts as the coupling factor between user and designer optimization problems. We analyze first the single carrier case as a starting point, which we immediately extend to multi carriers. We next formulate the operator revenue maximization problem using the same framework. Numerical simulations illustrate the mechanism developed and show the convergence of power and price levels of the users.
\end{abstract}

\section{Introduction}

Many wireless systems and schemes such as Orthogonal Frequency Division Multiplexing (OFDM),

This work has been supported in part by Deutsche Telekom Laboratories and German National Science Foundation (DFG) project BO $1734 / 5-2$. cognitive radios [12], and femtocells have spectrum allocated over multiple orthogonal carriers. In this paper, we consider uplink resource allocation of multicarrrier systems with strategic users having a scalarparameterized logarithmic utility function. The users decide independently on their power levels without revealing their utility functions, so as to maximize their individual utilities. The utility parameters may indicate the priority of the application, residual queue size etc. Concurrently, the base station has a social goal such as social welfare (sum of user utility) maximization which may not be achieved due to this strategic behavior of users. This is because at the Nash Equilibrium (NE) point of this noncooperative power control game there is misalignment of social objective and individual user objectives and this is known as Price of Anarchy. To counter this scenario, the base station acts as a mechanism designer and uses pricing schemes [9] to incentivise the users. We study distributed pricing algorithms in which the users decide on their power levels depending on their utility functions and the prices which are set by the designer.

The general setting considered here can be applied to cognitive radios, OFDM systems and femtocell systems with appropriate modifications such as additional constraints on interference or weighing the user utility functions. Cognitive radios having ability to sense their environment and high computational capacity can act strategically as independent decision makers [12]. The primary base station can act as the designer and employ pricing mechanisms to align power selection decision so as to achieve its global objective. Femtocell systems can be modeled as a Stackelberg game where a macro base station (MBS) act as the leader and multiple femto base stations (FBSs) as the followers [3].

An Iterative Water Filling (IWF) algorithm is pro- 
posed in [20] to maximise the sum rate in the presence of individual power constraints in a Gaussian Multiple Access Channel (GMAC). But this algorithm will converge to a non-pareto-optimal Nash equilibrium and inefficient sum rate when the independent and strategic users take their power levels in a distributed fashion. Pricing of transmit power for Pareto improvement of the inefficient Nash equilibrium in noncooperative power control game was introduced in [15]. The pricing function was linear in transmit power and the utility of users have the unit bits/Joule. A distributed pricing mechanism for interference coupled systems in which each user announces a price is proposed in [10]. The price signal from each user reflects the interference compensation price paid by the other users. In [17], a pricing based game for spectrum allocation with individual power constraint and multiple carriers is analyzed and a Price based Iterative Water Filling is proposed. The social optimization problem is taken as the weighted sum of Shannon capacities which are the utilities of individual users. To enable the users to achieve better Nash equilibria a price based iterative distributed water-filling algorithm is proposed in [17]. In [7], a modified Vicrey-Clarke-Groves (VCG) mechanism is obtained for allocation of a divisible resource in which the pricing function is modified for achieving social welfare and some other desirable properties. The price anticipating users have scalar parameterized logarithmic utility function and the designer centrally allocate the resource knowing the shape of the utility functions. The work in this paper is an extension of the work in [2] for a scalar parameterized logarithmic user utility functions which is unknown to the designer and individual user power constraint. We first motivate our problem for obtaining the pricing mechanisms for single carrier systems with individual user power constraints. Then, the extension to multi carrier systems is done first only with individual user power constraints and then with additional global power constraint.

In addition to pricing user transmit powers for obtaining social goals, the designer may like to maximise the revenue obtained from these prices. We next introduce pricing mechanisms for designer revenue maximization which may lead to non optimal social welfare. Myerson [13] introduced optimal auctions in which the designer knowing the distribution of private values of players maximizes the expected revenue. In [8], for a wide-band wireless network that employs CDMA as the spectrum access mechanism, the revenue maximization problem is formulated as a Stackelberg game. The optimal prices are obtained for the Nash equilibrium points. For revenue maximization in a similar setting, suboptimal constant distributed pricing scheme is proposed in [14]. In [1], for a general delay network, a two-stage dynamic pricing-congestion game in which the service provider sets a price anticipating demand of users and users chose their flow vectors given the prices, is analyzed. An optimal revenue maximizing pricing is proposed for networks with several competing oligopolistic and the extent of inefficiency loss is lower bounded. In [16], a lower bound for the ratio between the revenue from flat entre fee pricing rule and maximum revenue possible is provided, which they refer to as the Price of Simplicity (PoS). A price discrimination scheme is also studied and Price of Simplicity is obtained for it. In this paper, we come up with revenue maximizing pricing mechanisms for interference coupled multi-carrier wireless systems by manipulating the Nash equilibrium with optimal prices.

We propose mechanisms for multicarrrier systems, where the designer without knowing users utility functions achieve two different designer objectives through appropriate pricing. The pricing mechanisms obtained here can be implemented through a distributed iterative algorithm rather than existing heuristic suboptimal or centralized algorithms. We investigate mechanisms in which the user utility functions are modeled as scalar parameterized logarithmic functions. The users do not report their utility functions and just decide on their own power level. The users are not considered to be price anticipating here because in a distributed network there is an information asymmetry between the users and the designer. The users do not know the action and utility function of other users or the nature of pricing function. Hence, they cannot anticipate the exact impact of their action on the pricing function. Therefore, they just adopt a best response strategy by taking the price given by the designer.

Contributions of this paper are:

1) Distributed pricing mechanisms for uplink power and spectrum allocation of multi carrier systems which maximize net user utilities, with designer not knowing the utility functions of users.

2) Combination of both individual and total power constraints in the constraint set.

3) Distributed pricing mechanism for designer revenue maximization in multi carrier systems.

The rest of the paper is organized as follows. The next section presents the underlying system model which is used throughout the paper. Subsequently, Section 3 analyses pricing mechanisms for single carrier systems with individual power constraints. The results are extended to multi carrier systems in Section 4. Section 5 investigates mechanisms for designer revenue maximization. Numerical simulations and their results are 
shown in Section 6. The paper concludes with remarks of Section 7.

\section{System Model}

Let us consider an uplink multiple access system with spectrum divided into $N$ orthogonal carriers shared among $K$ users. We assume the base station acts as a designer $\mathcal{D}$ who manages the resource sharing among the users. In this scenario, let us define a $K$ player strategic non-cooperative game, $\mathcal{G}$, where each player $i \in \mathcal{A}$ has a respective decision variable $x_{i}$ such that

$$
x=\left[x_{1}, \ldots, x_{K}\right] \in \mathcal{X} \subset \mathbb{R}^{K \times N},
$$

where $\mathcal{X}$ is the decision space of all users. Let

$x_{-i}=\left[x_{1}, \ldots, x_{i-1}, x_{i+1}, \ldots x_{K}\right] \in \mathcal{X}_{-i} \subset \mathbb{R}^{K-1 \times N}$,

be the profile of decision variable of users other than $i^{t h}$ player and $\mathcal{X}_{-i}$ is the respective decision space. This paper assumes vector decision variables and a compact and convex decision space. Due to the inherent coupling between the users, the decisions of users directly affect each other's performance as well as the aggregate allocation of limited resources.

Each user decides on the power level over the $N$ carriers. Therefore,

$$
x_{i}=\left[x_{i}^{(1)}, \ldots, x_{i}^{(N)}\right],
$$

where $x_{i}^{(n)}=h_{i}^{(n)} p_{i}^{(n)}$ denote the received power level over carrier $n$ as a product of uplink transmission power $p_{i}^{(n)}$ and channel loss $0<h_{i}^{(n)}<1$ of player $i$. If linear interference is assumed, then the signalto-interference ratio (SIR) of the received signal on channel $n$ is

$$
\gamma_{i}^{(n)}=\frac{x_{i}^{(n)}}{\sum_{j \neq i} x_{j}^{(n)}+\sigma},
$$

where $\sigma$ represents the background noise. Let us denote the interference at receiver for user $i$ over channel $n$ as $I_{i}^{(n)}=\sum_{j \neq i} x_{j}^{(n)}+\sigma$.

The preferences of the users are captured by utility functions and for multi-carrier wireless systems it is given by

$$
\sum_{n} U_{i}\left(\gamma_{i}^{(n)}(x)\right): \mathcal{X} \rightarrow \mathbb{R}, \quad \forall i \in \mathcal{A},
$$

which are usually chosen to be continuous and differentiable for analytical tractability. We assume that $U_{i}($.$) is any concave non-decreasing function.$

In this paper we model the user utilities as logarith- mic in SINR parameterized by a scalar value, i.e.,

$$
U_{i}\left(\gamma_{i}(x)\right)=\alpha_{i} \sum_{n} \log \left(\gamma_{i}^{(n)}(x)\right) .
$$

where $\alpha_{i}$ is an application-dependent parameter private to the users. We also consider a modified version of logarithmic utility,

$$
U_{i}\left(\gamma_{i}(x)\right)=\alpha_{i} \sum_{n} \log \left(1+\gamma_{i}^{(n)}(x)\right) .
$$

which corresponds to the Shannon capacity achieved by each user $i$ over channel $n$, given by

$$
R_{i}=\sum_{n} \log \left(1+\gamma_{i}^{(n)}(x)\right) .
$$

Therefore, the utility of each user in the second case can be also interpreted as scalar parameterized Shannon capacity.

The designer $\mathcal{D}$ devises a pricing mechanism $\mathcal{M}$, which can be represented by the mapping $\mathcal{M}: \mathcal{X} \rightarrow$ $\mathbb{R}^{K \times N}$, implemented by introducing incentives in the form of prices to the users. The latter can be formulated by adding it as a cost term such that the player $i$ has the cost function

$$
J_{i}(x)=c_{i}(x)-\sum_{n} U_{i}\left(\gamma_{i}^{(n)}(x)\right) .
$$

The designer imposes prices $P_{i}^{(n)}(x)$ per unit power over channel $n$ for user $i$ to align the strategic users with the global objective. The total payment by user $i$ is then $\sum_{n} x_{i}^{(n)} P_{i}^{n}(x)$ and the individual cost of users will be

$$
J_{i}(x)=\sum_{n}\left(x_{i}^{(n)} P_{i}^{n}(x)-U_{i}\left(\gamma_{i}^{(n)}(x)\right)\right) .
$$

The user objective is to solve the following individual optimization problem in the strategic game

$$
\min _{x_{i}} J_{i}(x),
$$

under the given constraints of the strategic game, and prices imposed by the designer.

The Nash equilibrium (NE) is a widely-accepted and useful solution concept in strategic games, where no player has an incentive to deviate from it while others play according to their NE strategies. The NE $x^{*}$ of the game $\mathcal{G}$ is formally defined as

$$
x_{i}^{*}:=\arg \min _{x_{i}} J_{i}\left(x_{i}, x_{-i}^{*}\right), \quad \forall i \in \mathcal{A},
$$

where $x_{-i}^{*}=\left[x_{1}^{*}, \ldots, x_{i-1}^{*}, x_{i+1}^{*}, \ldots, x_{N}^{*}\right]$. The NE is at the same time the intersection point of players' best responses obtained by solving user problems individually.

Similar to player preferences, the designer objec- 
tive, e.g. maximization of aggregate user utilities or social welfare, can be formulated using a smooth objective function $V$ for the designer:

$$
V\left(x, U_{i}\left(\gamma_{i}(x)\right), c_{i}(x)\right): \mathcal{X} \rightarrow \mathbb{R},
$$

where $c_{i}(x)$ are user-specific pricing terms and player utilities, respectively. Hence, the global optimization problem of the designer is simply $\max _{x} V\left(x, U_{i}(x), c_{i}(x)\right)$, which it solves indirectly by setting prices. In some cases, the objective function $V$ characterizes the desirability of an outcome $x$ from the designers perspective. In other cases when the designer objective is to satisfy certain minimum performance constraints such as users achieving certain quality-ofservice levels, the objective can be characterized by a region (a subset of the game domain $\mathcal{X}$ ). The main problem the designer has is that he has no information about the utility function of the users except that it belongs to a class of concave nondecreasing (in this case logarithmic) functions.

Definition 1. Efficient mechanisms: Efficient mechanisms maximize designer objective, i.e. they solve the problem $\max _{x} V\left(x, U_{i}(x), c_{i}(x)\right)$.

\section{Single Carrier Systems}

We start with single carrier wireless systems, with individual user power constraints. The designer objective is to achieve social welfare and design of pricing mechanisms for this purpose is considered here.

\subsection{Pricing Mechanism with Single Carrier and Individual Power Constraint:}

Let us consider a single carrier, $N=1$. Then, the received power level of $i^{t h}$ user $x_{i}=h_{i} p_{i}$ and the signal-to-interference ratio (SIR) of the received signal is

$$
\gamma_{i}=\frac{x_{i}}{I_{i}},
$$

where $I_{i}=\sum_{j \neq i} x_{j}+\sigma$. With the pricing mechanism in place, each player $i$ 's cost is given by

$$
J_{i}(x)=P_{i} x_{i}-U_{i}\left(\gamma_{i}(x)\right),
$$

which is strictly convex in $x_{i}$.

Thus, the user optimization problem with individual power constraints will be to find the power level which minimizes his individual cost, i.e.,

$$
\min _{x_{i}} J_{i}(x) .
$$

Consequently, the general condition for player best response obtained from first order derivative is

$$
P_{i}-\frac{d U_{i}}{d x_{i}}=0, \forall i \in \mathcal{A} .
$$

For the case of $U_{i}=\alpha_{i} \log \left(\gamma_{i}(x)\right)$, the user best response becomes

$$
x_{i}=\frac{\alpha_{i}}{P_{i}} .
$$

In the case of Shannon capacity, i.e., $U_{i}=$ $\alpha_{i} \log \left(1+\gamma_{i}(x)\right)$,

$$
\frac{\alpha_{i}}{\sum_{j} x_{j}+\sigma}=P_{i}
$$

and

$$
x_{i}=\max \left\{\frac{\alpha_{i}}{P_{i}}-I_{i}, 0\right\}
$$

It can be observed that using this utility function will result in a soft user admission control scheme.

The equation (7) can be also written in terms of individual SINR as,

$$
\frac{d U_{i}}{d \gamma_{i}}=\frac{P_{i}}{d U_{i} / d x_{i}}, \forall i \in \mathcal{A}
$$

Using equation (5), we obtain

$$
\frac{d U_{i}}{d \gamma_{i}}=P_{i} I_{i}, \forall i \in \mathcal{A} .
$$

In this section, the global objective of the designer aims to maximize sum of utilities of users while trying to limit the user power levels to $P_{\max }$. The designer $\mathcal{D}$ solves the constrained optimization problem

$$
\max _{x} V(x) \Leftrightarrow \max _{x} \sum_{i} U_{i}\left(\gamma_{i}(x)\right)
$$

such that

$$
\frac{x_{i}}{h_{i}} \leq P_{\max } \forall i .
$$

Thus, the Lagrangian function of designer optimization problem can be written as:

$$
L=\sum_{i} U_{i}\left(\gamma_{i}(x)\right)-\sum_{i} \lambda_{i}\left(\frac{x_{i}}{h_{i}}-P_{\max }\right) .
$$

The resulting KKT conditions are

$$
\begin{gathered}
\frac{d U_{i}}{d x_{i}}+\sum_{j \neq i} \frac{d U_{j}}{d x_{i}}-\frac{\lambda_{i}}{h_{i}}=0, \forall i \\
\lambda_{i}\left(\frac{x_{i}}{h_{i}}-P_{\text {max }}\right)=0, \forall i
\end{gathered}
$$

The equation (13) can be rewritten as,

$$
\frac{d U_{i}}{d x_{i}}+\sum_{j \neq i} \frac{d U_{j}}{d \gamma_{j}} \frac{d \gamma_{j}}{d x_{i}}-\frac{\lambda_{i}}{h_{i}}=0, \forall i
$$

For aligning both the user problems and the global 
objective of the base station the above equation can be rewritten by substituting from the user equations in (7),

$$
P_{i}-\sum_{j \neq i} \frac{d U_{j}}{d \gamma_{j}} \frac{x_{j}}{I_{j}^{2}}-\frac{\lambda_{i}}{h_{i}}=0, \forall i
$$

By knowing the structure of user cost function and by substituting from equation (11) the designer can obtain the prices by solving

$$
P_{i}-\sum_{j \neq i} \frac{P_{j} x_{j}}{I_{j}}-\frac{\lambda_{i}}{h_{i}}=0, \forall i
$$

So the designer decides on the prices to the users, based on which users take action on their power levels, such that both the user problems and designer problem are solved concurrently and in a distributed manner. In [18], the designer dictates on the allocation of the users along with the prices according to VCG mechanism to obtain a socially optimal Nash equilibrium point. Here, in this paper the prices align the received power levels $(x)$ to solve both the user problems and the designer problem.

Using equation (5),

$$
P_{i}=\sum_{j \neq i} \gamma_{j} P_{j}+\frac{\lambda_{i}}{h_{i}} \forall i .
$$

We can see that unlike Kelly mechanism [11] for separable utilities, the prices are not the Lagrange multipliers. The optimal prices for interference coupled systems are solution of a of linear program which has system parameters and Lagrange multipliers as coefficients. Note that the $\gamma$ 's are the receiver SINR can be measured at the base station. Using this side information, (17) can be written in matrix form as,

$$
A \cdot P=B \cdot L,
$$

where

$$
\begin{gathered}
A:=\left(\begin{array}{cccc}
1 & -\gamma_{2} & \cdots & -\gamma_{N} \\
-\gamma_{1} & 1 & \cdots & -\gamma_{N} \\
\vdots & & \ddots & \vdots \\
-\gamma_{1} & -\gamma_{2} & \cdots & 1
\end{array}\right), \\
B:=\left(\begin{array}{cccc}
\frac{1}{h_{1}} & 0 & \cdots & 0 \\
0 & \frac{1}{h_{2}} & \cdots & 0 \\
\vdots & & \ddots & \vdots \\
0 & 0 & \cdots & \frac{1}{h_{N}}
\end{array}\right)
\end{gathered}
$$

and $L=\left[\lambda_{1}, \ldots, \lambda_{N}\right]^{T}$.

The designer assumes general concave function of SINR as utilities for users and therefore global ob- jective may not be always convex in the vector $x$. When the global optimization problem is not convex, the above solution may lead to a local optimum. But in the case of $U_{i}=\alpha_{i} \log \left(\gamma_{i}(x)\right)$ using Geometric Programming (GP) [6] or using the transformation given in [4] the global objective can be convexified. When users have utility function $U_{i}=\alpha_{i} \log \left(1+\gamma_{i}(x)\right)$, the global objective cannot be convexified using standard techniques like Geometric Programming (GP) [6]. A heuristic iterative method using Sigmoidal Programming (SP) was proposed in [6], to bring the solution in this case to near optimum.

\subsection{Iterative Distributed Algorithm}

We propose a Gradient update iterative distributed algorithm similar to the one in [5] to implement the pricing mechanism obtained above. A simple greedy best response will result in fluctuations of power levels and thus the users adopt a gradient best response for convergence purposes. From the alignment of user and designer problem we come up with an iterative method which uses the dual variables Lagrange multipliers $\lambda_{i}$ 's to converge to the optimal prices and power levels. The iterative pricing mechanism $\mathcal{M}^{a}$ for the logarithmic utility case is defined as

$$
\begin{gathered}
P(k+1)=(A)^{-1} B \cdot L(k), \\
p_{i}(k+1)=p_{i}(k)-\frac{\kappa_{i}}{h_{i}} \frac{\partial J_{i}}{\partial x_{i}} \quad\left(x_{i}\right) \quad \forall i \in \mathcal{A}, \\
\lambda_{i}(k+1)=\lambda_{i}(k)+\kappa_{D}\left(p_{i}(k+1)-P_{\max }\right),
\end{gathered}
$$

In the iterative process a search is carried out in $\lambda$ space to converge the power levels to an optimal point.

\section{Multi-Carrier Systems}

We consider a multi-carrier system, where the transmit power is allocated across multiple orthogonal channels as in OFDM. Each user receives a different price for power consumption over different channels and the prices influence the user best responses (IWF solution) [19]. In this section we extend the results obtained earlier for single carrier systems to multiple carrier systems.

\subsection{Pricing Mechanism with Multiple Carri- ers and Individual Power Constraint:}

For multi-carrier systems the user optimization problem from equation (3) will be,

$$
\max _{x_{i}} \sum_{n}\left(U_{i}\left(\gamma_{i}^{(n)}(x)\right)-x_{i}^{(n)} P_{i}^{(n)}\right) .
$$


The user best response obtained from first order derivative is

$$
\frac{d U_{i}\left(\gamma_{i}^{(n)}(x)\right)}{d x_{i}^{(n)}}-P_{i}^{(n)}=0, \forall i \in \mathcal{A}, n .
$$

and similar to equation (12),

$$
\frac{d U_{i}}{d \gamma_{i}^{(n)}}=P_{i}^{(n)} I_{i}^{(n)}, \forall i \in \mathcal{A}, n .
$$

For the case of

$$
\begin{gathered}
U_{i}(x)=\alpha_{i} \sum_{n} \log \left(\gamma_{i}^{(n)}\right), \forall i \in \mathcal{A}, \\
x_{i}^{(n)}=\frac{\alpha_{i}}{P_{i}^{(n)}} \forall i \in \mathcal{A}, n .
\end{gathered}
$$

When the utility of each user is taken as,

$$
\begin{array}{r}
U_{i}(x)=\alpha_{i} \sum_{n} \log \left(1+\gamma_{i}^{(n)}\right), \forall i \in \mathcal{A}, \\
x_{i}^{(n)}=\max \left\{\frac{\alpha_{i}}{P_{i}^{(n)}}-I_{i}^{(n)}, 0\right\} \forall i \in \mathcal{A}, n .
\end{array}
$$

The power allocation in the second case will result in an admission control over each channel and a waterfilling solution which will be now modified by the individual prices over the different channels.

The global objective of designer in multi-carrier systems with individual user power constraint is to solve the optimization,

$$
\max _{x} \sum_{i} \sum_{n}\left(U_{i}\left(\gamma_{i}^{(n)}(x)\right)\right.
$$

subject to

$$
\sum_{n} \frac{x_{i}^{(n)}}{h_{i}^{(n)}} \leq P_{\max } . \forall i, n
$$

So the Lagrangian function of designer can be written as:

$$
L=U_{i}(x)+\sum_{j \neq i} U_{j}(x)-\sum_{i} \lambda_{i}\left(\sum_{n} \frac{x_{i}^{(n)}}{h_{i}^{(n)}}-P_{\max }\right), \forall i, n
$$

where $\lambda_{i}$ 's are Lagrangian multipliers. The KarushKuhn-Tucker (K.K.T) conditions are given by:

$$
\begin{gathered}
\frac{d U_{i}\left(\gamma_{i}^{(n)}(x)\right)}{d x_{i}^{(n)}}+\sum_{j \neq i} \frac{d U_{j}\left(\gamma_{j}^{(n)}(x)\right)}{d x_{i}^{(n)}}-\frac{\lambda_{i}}{h_{i}^{(n)}}=0, \forall i, n, \\
\lambda_{i}\left(\sum_{n} \frac{x_{i}^{(n)}}{h_{i}^{(n)}}-P_{\max }\right)=0, \forall i, n \\
\sum_{n} \frac{x_{i}^{(n)}}{h_{i}^{(n)}} \leq P_{\max } \forall i, n .
\end{gathered}
$$

The equation (29) can be rewritten as,

$$
\frac{d U_{i}}{d x_{i}^{(n)}}+\sum_{j \neq i} \frac{d U_{j}}{d \gamma_{j}^{(n)}} \frac{d \gamma_{j}^{(n)}}{d x_{i}^{(n)}}-\frac{\lambda_{i}}{h_{i}^{(n)}}=0, \forall i, n .
$$

Aligning both the user problems and the global objective of the base station by substituting from the user equations in (24), the above equation becomes

$$
P_{i}^{(n)}-\sum_{j \neq i} \frac{d U_{j}^{(n)}}{d \gamma_{j}^{(n)}} \frac{x_{j}^{(n)}}{\left(I_{j}^{(n)}\right)^{2}}-\frac{\lambda_{i}}{h_{i}^{(n)}}=0, \forall i, n .
$$

By knowing the structure of user cost function and using (25) the designer can obtain the prices by solving

$$
\begin{gathered}
P_{i}^{(n)}-\sum_{j \neq i} \frac{P_{j}^{(n)} x_{j}^{(n)}}{I_{j}^{(n)}}-\frac{\lambda_{i}}{h_{i}^{(n)}}=0, \forall i, n \\
P_{i}^{(n)}=\sum_{j \neq i} \gamma_{j}^{n} P_{j}^{n}+\frac{\lambda_{i}}{h_{i}^{(n)}}, \forall i, n .
\end{gathered}
$$

The above system of equations can be written in matrix form as,

$$
A^{(n)} \cdot P^{(n)}=B^{(n)} \cdot L, \forall n,
$$

where $A^{(n)}$ and $B^{(n)}$ are defined accordingly.

4.1.1. Iterative Distributed Algorithm for MultiCarrier Systems. We now define pricing mechanism $\mathcal{M}^{b}$, for which the prices and bids from user for each carrier can be obtained using iterative methods as following.

$$
\begin{gathered}
P^{(n)}(k+1)=\left(A^{(n)}\right)^{-1} B^{(n)} \cdot L(k), \forall n \\
p_{i}^{(n)}(k+1)=p_{i}^{(n)}(k)-\frac{\kappa}{i}_{h_{i}^{(n)}}{\frac{\partial J_{i}}{\partial x_{i}^{(n)}}}^{-1}\left(x_{i}^{(n)}\right) \quad \forall i \in \mathcal{A}, \\
\lambda_{i}(k+1)=\lambda_{i}(k)+\kappa_{D}\left(\sum_{n} p_{i}^{(n)}(k+1)-P_{\text {max }}\right),
\end{gathered}
$$

Since the designer optimization problem can be convexified and thus admits a unique solution, we can find unique $\lambda$ 's which align it to the user convex optimization problems. Hence, there exist corresponding prices, obtained from the matrix transformation given in (33), which will determine the optimal power levels.

The users do not have any incentive to select their power levels based on values other than their true $\alpha$ 's because the pricing mechanism solves their individual optimization problems using correct values of $\alpha$ 's indirectly through observing their actions. Since they cannot exactly quantify the impact of their action on the prices set by the designer, they will always take 
the prices and employ best response based on that The algorithm which also shows the information flow for the iterative method is given below in Algorithm 1.

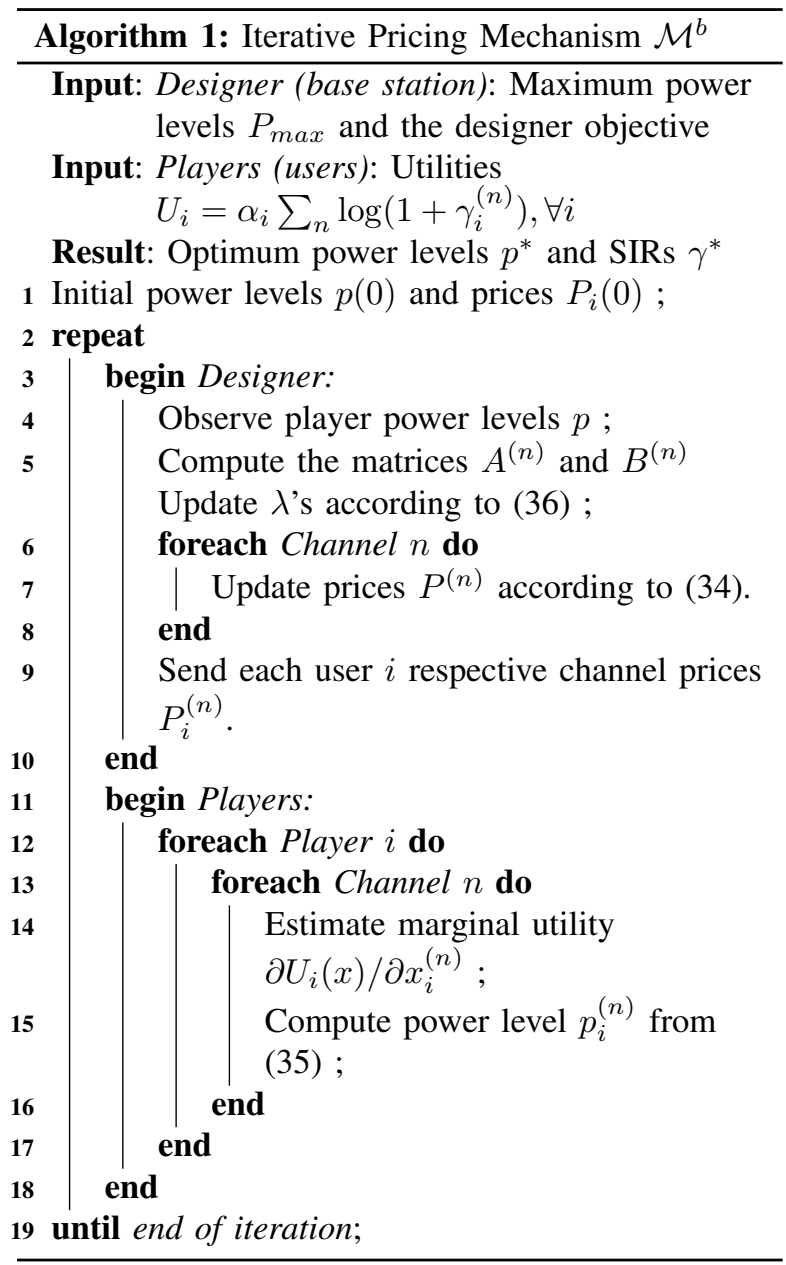

\subsection{Pricing Mechanism with Multiple Car- riers and Individual and Total Power Con- straints:}

Due to interference considerations in the cell, the total power transmitted by the users is to be kept below a threshhold. Now we modify the global optimization problem of designer in the previous section with total power constraint on top of individual user power constraint. So the global optimization problem in this case is,

$$
\max _{x} \sum_{i} U_{i}(x)
$$

subject to

$$
\sum_{n} \frac{x_{i}^{(n)}}{h_{i}^{(n)}} \leq P_{\max } \forall i
$$

and

$$
\sum_{i} \sum_{n} \frac{x_{i}^{(n)}}{h_{i}^{(n)}} \leq P_{\text {total }},
$$

where $P_{\text {total }}$ is the total power limit. So the Lagrangian function of designer can be written as:

$$
\begin{aligned}
L=V(x) & -\sum_{i} \lambda_{i}\left(\sum_{n} \frac{x_{i}^{(n)}}{h_{i}^{(n)}}-P_{\max }\right) \\
& -\lambda\left(\sum_{i} \sum_{n} \frac{x_{i}^{(n)}}{h_{i}^{(n)}}-P_{\text {total }}\right),
\end{aligned}
$$

where $\lambda_{i}$ 's and $\lambda$ are Lagrangian multipliers. The KKT conditions are given by:

$$
\begin{gathered}
\frac{d U_{i}}{d x_{i}^{(n)}}+\sum_{j \neq i} \frac{d U_{j}(x)}{d x_{i}^{(n)}}-\frac{\lambda_{i}}{h_{i}^{(n)}}-\frac{\lambda}{h_{i}^{(n)}}=0, \forall n, \\
\lambda_{i}\left(\sum_{n} \frac{x_{i}^{(n)}}{h_{i}^{(n)}}-P_{\max }\right)=0, \forall i, \\
\lambda\left(\sum_{i} \sum_{n} \frac{x_{i}^{(n)}}{h_{i}^{(n)}}-P_{\text {total }}\right)=0 .
\end{gathered}
$$

By using equation(24) to align the solution of both problems, we have:

$$
P_{i}^{(n)}=-\sum_{j \neq i} \frac{d U_{j}(x)}{d x_{i}^{n}}+\frac{\lambda_{i}}{h_{i}^{(n)}}+\frac{\lambda}{h_{i}^{(n)}} \forall i, n
$$

Using (27),

$$
\begin{gathered}
P_{i}^{(n)}=\sum_{j \neq i} \frac{x_{j}^{n} P_{j}^{n}}{\left(\sum_{k \neq j} x_{k}^{n}+\sigma\right)}+\frac{\lambda_{i}}{h_{i}^{(n)}}+\frac{\lambda}{h_{i}^{(n)}}, \forall i, n, \\
P_{i}^{(n)}=\sum_{j \neq i} \gamma_{j}^{n}\left(P_{j}^{n}\right)+\frac{\lambda_{i}}{h_{i}^{(n)}}+\frac{\lambda}{h_{i}^{(n)}}, \forall i, n .
\end{gathered}
$$

It can be written in matrix form as,

$$
A^{(n)} \cdot P^{(n)}=B^{(n)} \cdot L_{t}, \forall n,
$$

where $A^{(n)}$ and $B^{(n)}$ are defined accordingly and $L_{t}=\left[\lambda_{1}, \ldots, \lambda_{N}, \lambda\right]^{T}$. The prices and bids from user for each carrier can be obtained using iterative pricing mechanism $\mathcal{M}_{c}$ as following.

$$
\begin{gathered}
P^{(n)}(k+1)=(A)^{(n)^{-1}} B^{(n)} \cdot L(k), \forall n \\
p_{i}^{(n)}(k+1)=p_{i}^{(n)}(k)-\frac{\kappa_{i}}{h_{i}^{(n)}}{\frac{\partial J_{i}}{\partial x_{i}^{(n)}}}^{-1}\left(x_{i}^{(n)}\right) \forall i \in \mathcal{A},
\end{gathered}
$$


$\lambda_{i}(k+1)=\lambda_{i}(k)+\kappa_{D}\left(\sum_{n} p_{i}^{(n)}(k+1)-P_{\max }\right), \forall i \in \mathcal{A}$

and

$\lambda(k+1)=\lambda(k)+\kappa_{D}\left(\sum_{i} \sum_{n} p_{i}^{(n)}(k+1)-P_{\text {total }}\right)$.

The iterative algorithm $\mathcal{M}_{c}$ can be implemented similar to as demonstrated above in Algorithm 1.

\section{Pricing Mechanism for Designer Rev- enue Maximization}

In many practical scenarios the designer will be interested more in maximizing her revenue than sum of utilities of users. In this section, the global objective of the designer aims to maximize her total revenue as a monopolistic entity, while trying to limit the user power levels to $P_{\max }$. The total revenue of the designer will be,

$$
V(x)=\sum_{j} \sum_{n} P_{j}^{(n)}(x) x_{j}^{(n)} .
$$

The designer $\mathcal{D}$ solves the constrained optimization problem

$$
\max _{x} V(x) \text { such that } \sum_{n} \frac{x_{i}^{(n)}}{h_{i}^{(n)}} \leq P_{\max } \forall i, n .
$$

and

$$
\sum_{j} \sum_{n} \frac{x_{j}^{(n)}}{h_{j}^{(n)}} \leq P_{\text {total }} .
$$

By substituting equation (24), we obtain

$$
V(x)=\sum_{j} \sum_{n} \frac{d U_{j}}{d x_{j}^{(n)}} x_{j}^{(n)},
$$

and this objective function is not guaranteed to be convex. To find a local maximum we form the Lagrange function given by

$$
\begin{array}{r}
L=V(x)-\sum_{j} \mu_{j}\left(\sum_{n} \frac{x_{j}^{(n)}}{h_{j}^{(n)}}-P_{\max }\right) \\
+\mu \sum_{j} \sum_{n} \frac{x_{j}^{(n)}}{h_{j}^{(n)}}-P_{\text {total }},
\end{array}
$$

where $\mu_{i}$ 's and $\mu$ are Lagrange multipliers. The resulting KKT conditions for optimality are

$$
P_{i}^{(n)}+\sum_{j} x_{j}^{(n)} \frac{d^{2} U_{j}^{(n)}}{d x_{i}^{(n)} d x_{j}^{(n)}}-\mu_{i}-\mu=0, \forall i, n .
$$

$$
\begin{gathered}
\mu_{i}\left(\sum_{n} \frac{x_{i}^{(n)}}{h_{i}^{(n)}}-P_{\max }\right)=0, \forall i \\
\mu\left(\sum_{j} \sum_{n} \frac{x_{j}^{(n)}}{h_{j}^{(n)}}-P_{\text {total }}\right)=0
\end{gathered}
$$

Let us analyze this problem for the wireless channel example,

$$
U_{i}(x)=\alpha_{i} \sum_{n} \log \left(1+\gamma_{i}^{(n)}\right) .
$$

Substituting from equation (27), the above equation becomes

$$
P_{i}^{(n)}-\sum_{j} \frac{x_{j}^{(n)} \alpha_{j}}{\left(\sum_{k} x_{k}^{(n)}+\sigma\right)^{2}}-\frac{\mu_{i}}{h_{i}^{(n)}}-\frac{\mu}{h_{i}^{(n)}}=0, \forall i, n .
$$

By aligning the problems we can rewrite it as,

$$
P_{i}^{(n)}-\sum_{j} \frac{x_{j}^{(n)} P_{j}^{(n)}}{\left(\sum_{k} x_{k}^{(n)}+\sigma\right)}-\frac{\mu_{i}}{h_{i}^{(n)}}-\frac{\mu}{h_{i}^{(n)}}=0, \forall i, n
$$

Let us denote $F_{j}^{(n)}=\frac{x_{j}^{(n)}}{\left(\sum_{k} x_{k}^{(n)}+\sigma\right)}$, and note that $F_{j}^{(n)}=\frac{\gamma_{i}^{(n)}}{1+\gamma_{i}^{(n)}}$ is already available as side information. Then, the above equation can be again rewritten as

$$
P_{i}^{(n)}-\sum_{j} F_{j}^{(n)} P_{j}^{(n)}=\frac{\mu_{i}}{h_{i}^{(n)}}+\frac{\mu}{h_{i}^{(n)}}, \forall i, n .
$$

As in previous cases we obtain a matrix form as,

$$
C^{(n)} \cdot P^{(n)}=D^{(n)} \cdot K,
$$

where

$$
C^{(n)}:=\left(\begin{array}{cccc}
1+F_{1}^{(n)} & -F_{2}^{(n)} & \cdots & -F_{N}^{(n)} \\
-F_{1}^{(n)} & 1+F_{2}^{(n)} & \cdots & -F_{N}^{(n)} \\
\vdots & & \ddots & \vdots \\
-F_{1}^{(n)} & -F_{2}^{(n)} & \cdots & 1+F_{N}^{(n)}
\end{array}\right)
$$

and

$$
D^{(n)}:=\left(\begin{array}{ccccc}
\frac{1}{h_{1}^{(n)}} & 0 & \cdots & 0 & \frac{1}{h_{1}^{(n)}} \\
0 & \frac{1}{h_{2}^{(n)}} & \cdots & 0 & \frac{1}{h_{2}^{(n)}} \\
\vdots & & \ddots & \vdots & \\
0 & 0 & \cdots & \frac{1}{h_{N}^{(n)}} & \frac{1}{h_{N}^{(n)}}
\end{array}\right),
$$

and $K=\left[\mu_{1}, \ldots, \mu_{N}, \mu\right]^{T}$.

An iterative method which uses Lagrangian, $\mu_{i}$ 's and $\mu$ can give the prices and powers. For this we define now an iterative pricing mechanism $\mathcal{M}^{d}$ as similar to $\mathcal{M}^{c}$ in previous section.

$$
P^{(n)}(k+1)=\left(C^{(n)}\right)^{-1} D^{(n)} \cdot K(k),
$$




$$
\begin{aligned}
& p_{i}^{(n)}(k+1)=p_{i}(k)-\frac{\kappa}{i}_{h_{i}^{(n)}}{\frac{\partial J_{i}}{\partial x_{i}^{(n)}}}^{-1}\left(x_{i}^{(n)}\right) \forall i \in \mathcal{A}, n, \\
& \mu_{i}(k+1)=\mu_{i}(k)+\kappa_{D}\left(\sum_{n} p_{i}^{(n)}(k+1)-P_{\max }\right), \quad \forall i \in \mathcal{A},
\end{aligned}
$$

and

$\mu(k+1)=\mu(k)+\kappa_{D}\left(\sum_{i} \sum_{n} p_{i}^{(n)}(k+1)-P_{\text {total }}\right)$.

\section{Simulations}

In this section, numerical simulation results are presented for the case, $U_{i}(x)=\alpha_{i} \sum_{n} \log \left(1+\gamma_{i}^{(n)}\right)$, to establish the efficiency and convergence of the proposed mechanisms. First, the iterative pricing mechanism for single carrier systems is illustrated numerically. We simulate this scenario with 10 users and the following arbitrarily chosen utility parameters

$$
\alpha=[0.23,1.33,0.73,0.28,1.13,1.65,
$$

The users update their power levels according to (22) at each time step $k \geq 1$ with a step size of $\kappa=0.02$. The designer, on the other hand, updates the Lagrangian multipliers $\lambda$ 's and price vector $P$ based on (23) and (21), where $P_{\max }=1$ and $\kappa_{D}=0.02$. The background noise parameter is $\sigma=0.5$. The convergence of the mechanism $\mathcal{M}^{a}$ is depicted in Figures 1 and 2. It is observed that some of users with higher value of utility parameters get the full power and other users are not allocated with any power, resulting in an admission control scheme as expected.

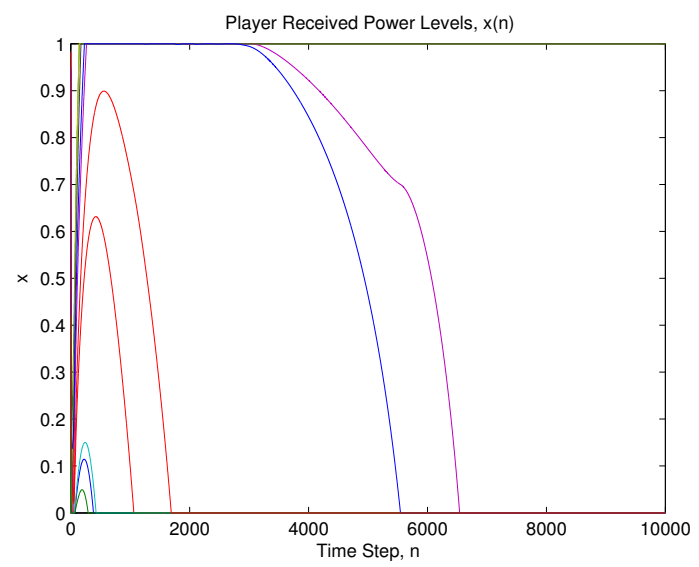

Figure 1. The evolution of user power levels $x$ in pricing mechanism $\mathcal{M}_{a}$ for a single carrier.

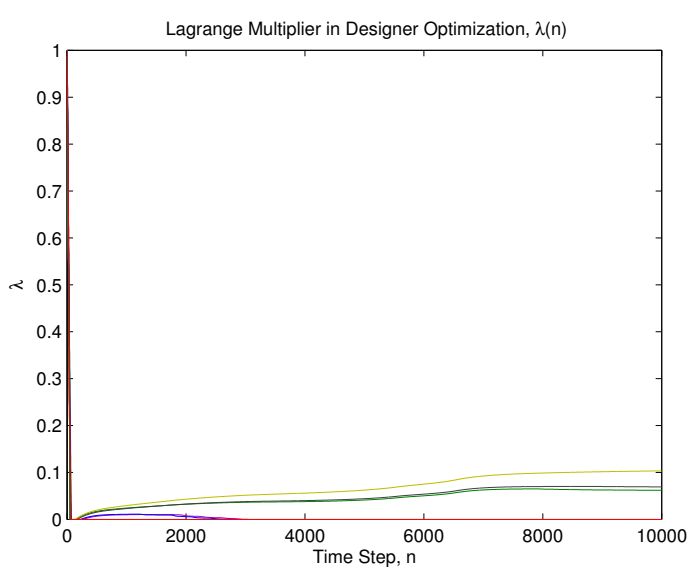

Figure 2. The evolution of Lagrange multiplier $\lambda$ in pricing mechanism $\mathcal{M}_{a}$ for a single carrier.

Next, the convergence of the iterative pricing mechanism with additional total power constraint is illustrated numerically in Figures 3 and 4. The total power constraint $P_{\text {total }}=2$. We observed solution boundary which can be seen as a virtual admission control.

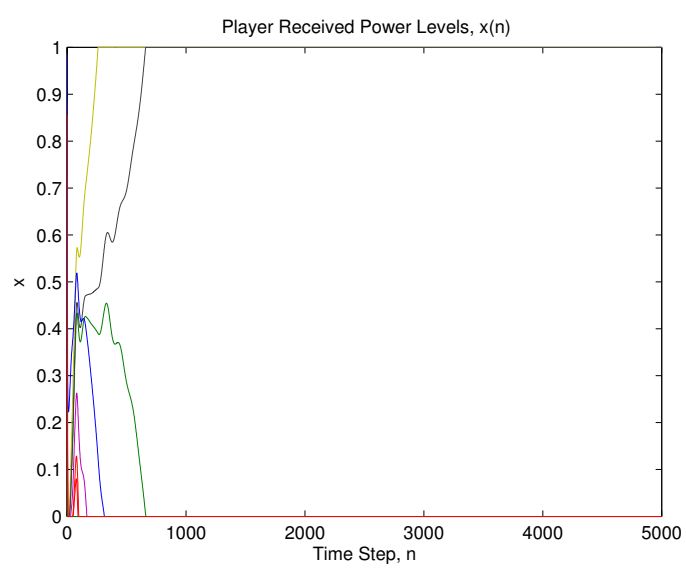

Figure 3. The evolution of user power levels $x$ in pricing mechanism with total power constraint.

The power levels for multi carrier system with number of carriers $M=5$ and number of users $N=10$ are plotted in Figure 5. The other parameters are same as above. For demonstration purpose the curves are shown for 3 users.

Finally, for the revenue maximizing mechanism, the convergence of power levels and lambda levels are plotted in Figures 6 and 7. A boundary solution behavior is observed as similar to efficiency maximizing mechanisms, but with different number of users touching the power constraint. 


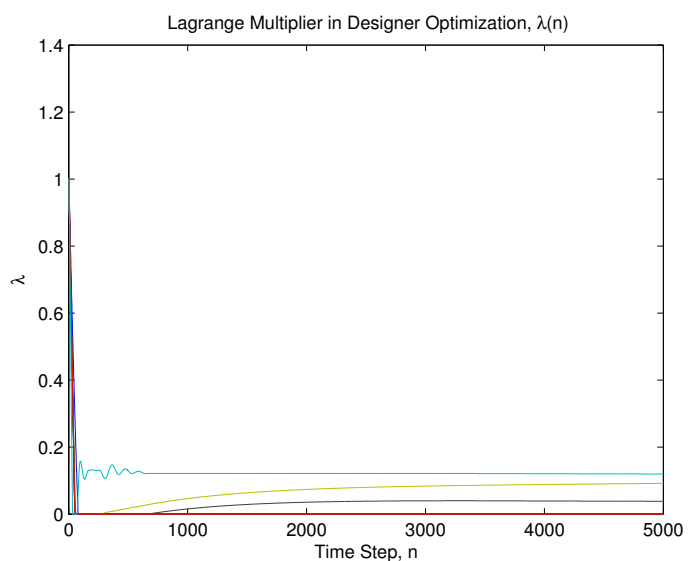

Figure 4. The evolution of Lagrange multiplier $\lambda$ in pricing mechanism with total power constraint.

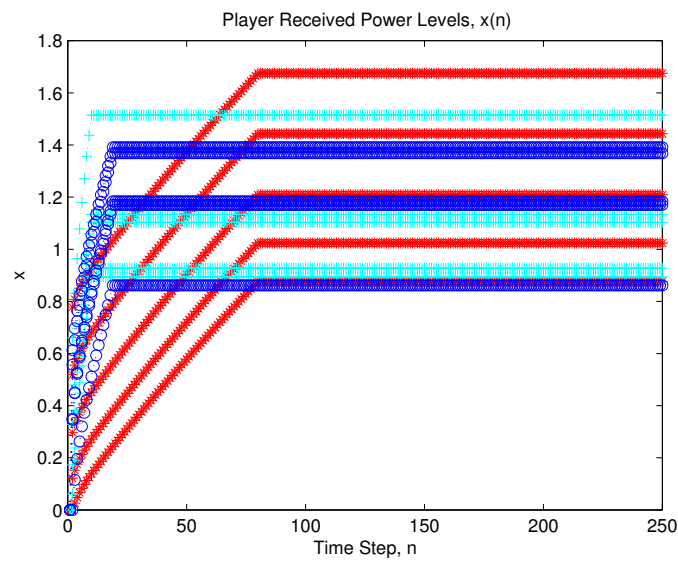

Figure 5. The evolution of user power levels $x$ in pricing mechanism for multiple carriers.

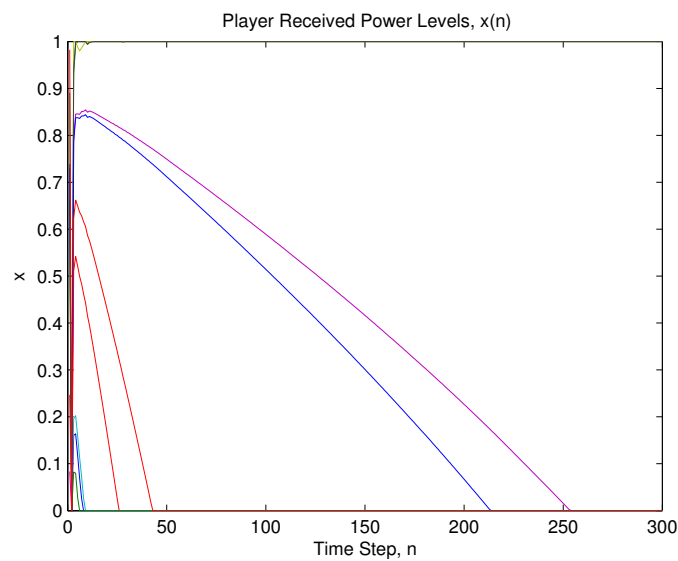

Figure 6 . The evolution of user power levels $x$ in pricing mechanism $\mathcal{M}_{d}$ for revenue maximization.

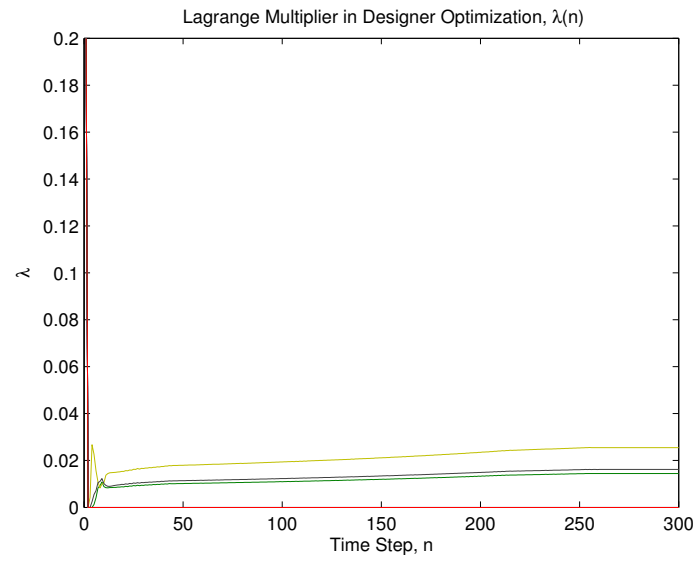

Figure 7. The evolution of Lagrange multiplier $\lambda$ in pricing mechanism $\mathcal{M}_{d}$ for revenue maximization.

\section{Conclusions}

In this paper, an uplink power control game of multi-carrier wireless systems with strategic users is analyzed. The users have scalar-parameterized logarithmic utility functions that are unknown to the designer (base station). Distributed and iterative pricing mechanisms for uplink power and spectrum allocation are proposed for two different designer objectives: social welfare and revenue maximization. First, pricing mechanisms which maximize social welfare for single carrier systems with users having individual power constraints are analyzed. We observed that the prices are a linear transformation of the Lagrange multipliers, and align user optimization problems with the designer problem. The iterative algorithm is observed to converge to unique optimal power and price levels in numerical simulations. Simulation results depicting the convergence of power levels show that some of users with higher value of utility parameter get the full power and others are allocated with no power resulting in an admission control. Next, the results are generalized to a case where users power levels are spread over multiple carriers. Then, on top of individual power constraints, total power constraint is added to the constraint set. Finally, iterative pricing mechanism for designer revenue maximization is also analyzed for multi carrier systems.

The future directions include generalizing these results to the case where users have general concave utility functions and analyzing the convergence of gradient update iterative algorithms. 


\section{References}

[1] D. Acemoglu and A. Ozdaglar, "Competition and efficiency in congested markets," Mathematics of Operations Research, vol. 32, no. 1, pp. 1-31, 2007.

[2] T. Alpcan and L. Pavel, "Nash Equilibrium Design and Optimization," in Proc. of Intl. Conf. on Game Theory for Networks (GameNets 2009), Istanbul, Turkey, May 2009.

[3] M. Bennis and M. Debbah, "On spectrum sharing with underlaid femtocell networks," in Second IEEE Workshop on Indoor and Outdoor Femto Cells (in conjunction with IEEE PIMRC'10), Istanbul, Istanbul, Turkey, 2010.

[4] H. Boche and M. Schubert, "A Superlinearly and Globally Convergent Algorithm for Power Control and Resource Allocation With General Interference Functions," IEEE/ACM Transactions on Networking, vol. 16, no. 2, pp. 383-395, April 2008.

[5] M. Chiang, "Balancing transport and physical layers in wireless multihop networks: jointly optimal congestion control and power control," IEEE Journal on Selected Areas in Communications, vol. 23, no. 1, pp. 104-116, 2005.

[6] _ - "Geometric programming for communication systems," Commun. Inf. Theory, vol. 2, pp. 1-154, July 2005. [Online]. Available: http://portal.acm.org/ citation.cfm?id=1166381.1166382

[7] A. K. Chorppath, S. Bhashyam, and R. Sundaresan, "A convex optimization framework for almost budget balanced allocation of a divisible good," IEEE Transactions on Automation Science and Engineering, 2011.

[8] A. A. Daoud, T. Alpcan, S.Agarwal, and M. Alanyali, "A stackelberg game for pricing uplink power in wideband cognitive radio networks," in 47th IEEE Conference on Decision and Control, 2008. CDC 2008., 9-11 December 2008, pp. 1422 - 1427.

[9] J. Hirshleifer, A. Glazer, and D. Hirshleifer, Price Theory and Applications Decisions, Markets, and Information. Cambridge, U.K.: Cambridge Univ. Press, 2005.

[10] J. Huang, R. Berry, and M. Honig, "Distributed Interference Compensation for Wireless Networks," IEEE Journal on Selected Areas in Communications, vol. 24, no. 5, pp. 1074-1084, May 2006.

[11] F. P. Kelly, A. K. Maulloo, and D. Tan, "Rate control in communication networks: shadow prices, proportional fairness and stability," Journal of the Operational Research Society, vol. 49, pp. 237-252, 1998.

[12] J. Mitola, "Cognitive radio for flexible mobile multimedia communications," Mobile Networks and Applications, , no. 6, pp. 435-441, 2001.
[13] R. B. Myerson, "Optimal auction design," Mathematics of Operations Research, vol. 6, no. 1, pp. 58-73, 1981.

[14] S. Ren and M. van der Schaar, "Revenue maximization and distributed power allocation in cognitive radio networks," in Proceedings of the 2009 ACM workshop on Cognitive radio networks, ser. CoRoNet '09. New York, NY, USA: ACM, 2009, pp. 43-48. [Online]. Available: http://doi.acm.org/10.1145/1614235.1614246

[15] C. Saraydar, N. Mandayam, and D. Goodman, "Efficient power control via pricing in wireless data networks," IEEE Transactions on Communications, vol. 50, no. 2, pp. 291-303, Feb. 2002.

[16] S. Shakkottai, R. Srikant, A. Ozdaglar, and D. Acemoglu, "The price of simplicity," in Forty-First Asilomar Conference on Signals, Systems and Computers, ACSSC 2007, Pacific Grove, CA, 2007, pp. 1450 1454.

[17] F. Wang, M. Krunz, and S. Cui, "Price-based spectrum management in cognitive radio networks," IEEE Journal of Selected Topics in Signal Processing, vol. 2, no. 1, pp. 74-87, February 2008.

[18] S. Yang and B. Hajek, "VCG-Kelly mechanisms for allocation of divisible goods: Adapting VCG mechanisms to one-dimensional signals," in 40th Annual Conference on Information Sciences and Systems, Princeton, New Jersey, 2006, pp. 1391-1396.

[19] D. D. Yu and J. M. Cioffi, "Iterative water-filling for optimal resource allocation in OFDM multiple-access and broadcast channels," in IEEE Global Telecommunications Conference (GLOBECOM '06), San Francisco, CA, 2006.

[20] W. Yu, W. Rhee, S. Boyd, and J. M. Cioffi, "Iterative water-filling for gaussian vector multiple-access channels," IEEE Transactions on Information Theory, vol. 50, no. 1, pp. 145-152, 2004. 